PALEO

Revue d'archéologie préhistorique

$17 \mid 2005$

Varia

\title{
Réflexions sur la fonction des microgravettes et la question de l'utilisation de l'arc au gravettien ancien
}

Comments on the function of microgravettes and on the problem of the use of the bow in Early Gravettian times

Maureen Hays et Frédéric Surmely

(2) OpenEdition

Journals

Édition électronique

URL : http://journals.openedition.org/paleo/899

DOI : $10.4000 /$ paleo.899

ISSN : 2101-0420

Éditeur

SAMRA

Édition imprimée

Date de publication : 1 décembre 2005

Pagination : 145-156

ISSN : 1145-3370

Référence électronique

Maureen Hays et Frédéric Surmely, «Réflexions sur la fonction des microgravettes et la question de I'utilisation de l'arc au gravettien ancien », PALEO [En ligne], 17 | 2005, mis en ligne le 23 avril 2010 consulté le 07 juillet 2020. URL : http://journals.openedition.org/paleo/899 ; DOI : https://doi.org/ 10.4000/paleo.899

Ce document a été généré automatiquement le 7 juillet 2020

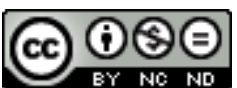

PALEO est mis à disposition selon les termes de la licence Creative Commons Attribution - Pas d'Utilisation Commerciale - Pas de Modification 4.0 International. 


\section{Réflexions sur la fonction des microgravettes et la question de l'utilisation de l'arc au gravettien ancien}

Comments on the function of microgravettes and on the problem of the use of the bow in Early Gravettian times

Maureen Hays et Frédéric Surmely

\section{NOTE DE L'ÉDITEUR}

La traduction du rapport d'analyse de M. Hays a été faite par C. Surmely.

Nous adressons nos chaleureux remerciements, pour les facilités données à cette étude, à Ph. Alix, J. Tournadre, A. Roubille, à la mairie de Mirefleurs, ainsi qu'à G. Marchesseau (musée du Périgord) et au personnel scientifique du musée d'histoire naturelle de La Rochelle. La fouille du gisement est financée par la DRAC Auvergne et la communauté de communes de Gergovie-Vald'Allier.

\section{Introduction}

1 Il y a bien longtemps que les archéologues s'intéressent à la fonction des microlithes. Pour les périodes de l'Épipaléolithique et du Mésolithique, des découvertes dans les sites du nord de l'Europe ont permis de montrer qu'il s'agissait d'éléments de flèches. Pour les périodes antérieures, les choses sont plus complexes, du fait de l'absence de témoins organiques, alors même que les microlithes sont très abondants dans la majorité des cultures du Paléolithique supérieur. Les progrès récents des techniques de 
fouilles, notamment par la mise en œuvre d'un tamisage à l'eau exhaustif des sédiments avec une maille fine, ont permis de constater l'importance quantitative des microlithes.

Depuis maintenant cinq ans, nous avons entrepris l'étude d'un gisement de plein air, situé au lieu-dit Le Sire, sur la commune de Mirefleurs (département du Puy-de-Dôme). A ce stade des recherches, le site présente deux niveaux du Gravettien ancien, datés d'environ 30000 BP (Surmely et al. 2003 et soumis). L'industrie lithique se caractérise par une très forte proportion de microlithes et de pointes à dos $(43 \%$ du total des outils), parmi lesquelles les pointes à dos de petite taille et les microgravettes figurent au premier rang. Nous nous sommes donc légitimement interrogés sur le type d'utilisation de ces outils. Pour ce faire, nous avons choisi, dans un premier temps, de prendre les mesures exactes des armatures. Ensuite, un examen tracéologique a été réalisé sur une sélection de pièces. Enfin, nous avons choisi, dans une perspective comparative, de faire une étude de flèches issues de collections ethnographiques provenant de différents continents, conservées dans deux musées français.

\section{Le gisement du Sire et les microgravettes}

3 Le Sire est un gisement de plein air qui se situe en Auvergne, dans la plaine de Limagne, sur le flanc d'une petite colline dominant la vallée de l'Allier. Le site offre une large vue sur la vallée, grand axe reliant l'Auvergne au Bassin Parisien, à proximité immédiate de gîtes riches en silex tertiaire. Les investigations archéologiques, qui ont débuté en 2000, ont révélé l'existence d'un campement très étendu, marqué par une abondance exceptionnelle de restes de chevaux (Surmely et al. 2003) et présentant au moins deux niveaux d'occupation. Les caractéristiques de l'industrie lithique et les datations $14 \mathrm{C}$ s'accordent pour placer l'occupation humaine durant le Gravettien ancien, période jusqu'à lors inconnue en Auvergne. L'outillage est constitué pour une large part de microlithes et de pointes ( $43 \%$ de l'ensemble des outils) (fig. 1). Il est à noter que l'industrie osseuse est totalement absente et que les objets de parure se limitent à quelques pièces. 
Figure 1 - Microgravettes du Sire.

Figure 1 - Microgravettes from the Sire site.
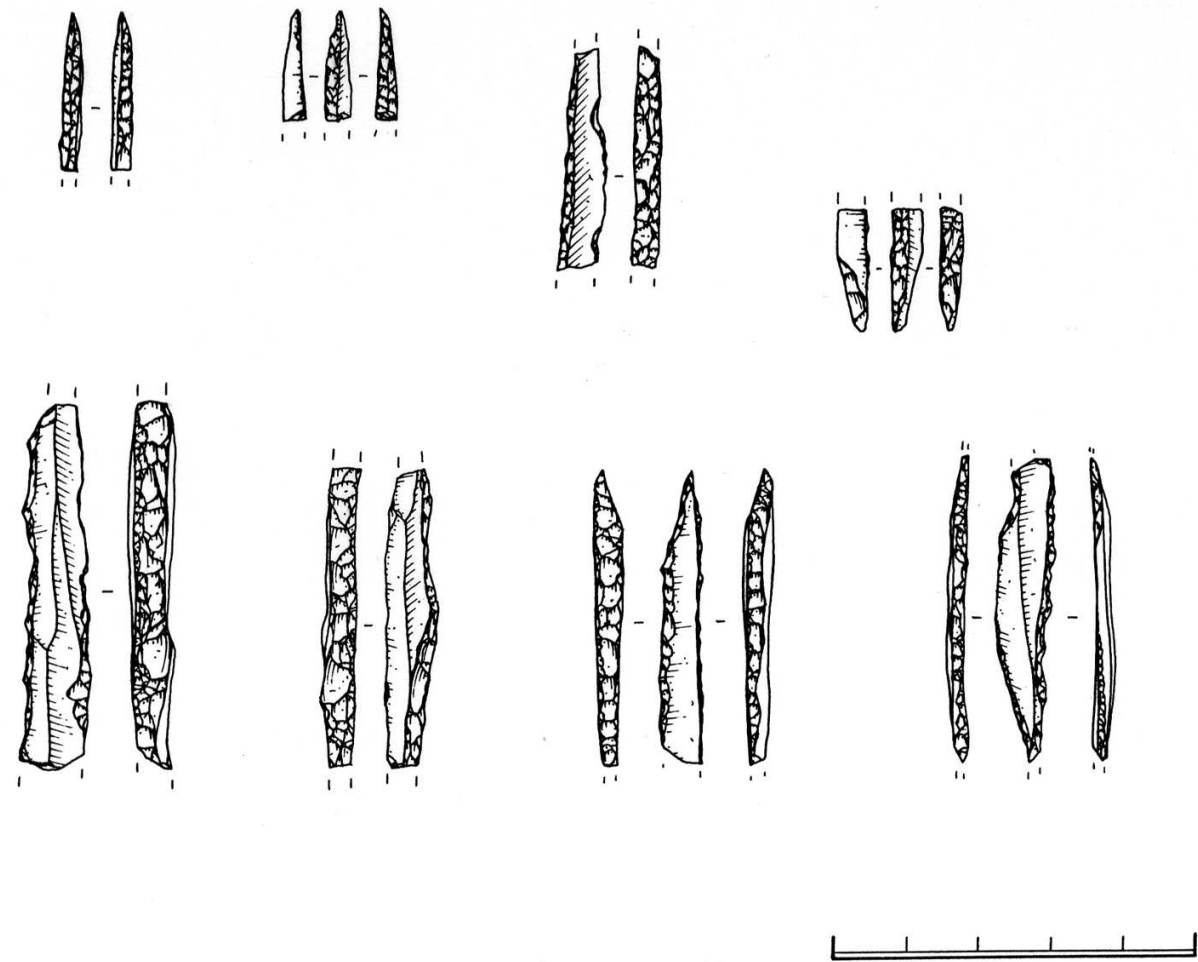

Dessins Ph. Alix.

Drawings Ph. Alix.

4 L'industrie lithique est majoritairement façonnée dans le silex tertiaire local. Mais une partie des pièces est issue de silex marins du Secondaire, dont l'origine est lointaine (150 à $250 \mathrm{~km}$ de distance selon les types utilisés; Surmely et Pasty 2003 ; Surmely et al. 2003), avec notamment un silex blond de la craie et un silex de l'Infralias, provenant vraisemblablement du secteur de Saint-Jeanvrin (Cher).

La définition typologique de la microgravette est variable d'un auteur à l'autre, ce qui est une gêne certaine pour les comparaisons (Digan 2001). L'acception traditionnelle (Sonneville-Bordes et Perrot 1956 ; Demars et Laurent 1989) est celle d'une pointe à dos rectiligne, aménagée sur une lamelle ou petite lame et portant une retouche, partielle ou continue, directe ou inverse, sur le bord opposé. Cette classification porte donc l'accent sur un caractère, la présence d'une retouche sur le bord opposé, mais laisse très flou le paramètre dimensionnel. Comme d'autres chercheurs (Digan 2001; O'Farrell 2004), nous avons préféré adopter une définition plus précise, en réservant l'emploi du terme de microgravette aux pièces aménagées sur lamelle, avec une largeur maximale de $8 \mathrm{~mm}$ (d'autres auteurs ont retenu la limite de $6 \mathrm{~mm}$, comme Nespoulet 1996). Les exemplaires de largeur supérieure, aménagés sur lame, ont été comptés parmi les pointes de La Gravette. Ceux qui sont dépourvus de retouche sur le bord opposé, comme pointes à dos. Enfin les pièces dépourvues de pointes ont été rangées dans la catégorie des lamelles à dos.

6 Il est à noter que nous avons retrouvé également un nombre important de fragments mésiaux d'armatures microlithiques qui correspondent vraisemblablement à des 
microgravettes. Mais, par précaution et par souci de rigueur, nous avons choisi de les classer parmi le groupe des « fragments d'armatures indéterminées ».

7 Avec ces critères typologiques, notre échantillon de 93 pièces se décompose en 32 microgravettes, 20 pointes à dos microlithiques, 6 pointes de la Gravette, 1 lamelle à dos et 34 armatures indéterminées.

Les microgravettes sont presque toutes brisées et ne se prêtent donc pas à la mesure de la longueur. Par contre, la mesure de la largeur est possible. Celle-ci s'échelonne entre 2,5 et $8 \mathrm{~mm}$, avec une moyenne de 4,9 $\mathrm{mm}$ (fig. 2). Cette valeur doit être considérée comme légèrement inférieure ${ }^{1}$ à la largeur réelle des pièces, compte tenu du fait que la mesure a été prise majoritairement sur des extrémités distales. Elle correspond aux valeurs relevées sur d'autres sites gravettiens plus récents, comme ceux de Rabier (Soriano 1998), de la Vigne Brun (Digan 2001) et du niveau 3 de l'abri Pataud (Nespoulet 1996).

Figure 2 - Distribution des largeurs des microgravettes et pointes de la Gravette du gisement du Sire (Mirefleurs, 63).

Figure 2 - Distribution of the microgravettes and gravette points from the Sire site (Mirefleurs 63) according to their width.

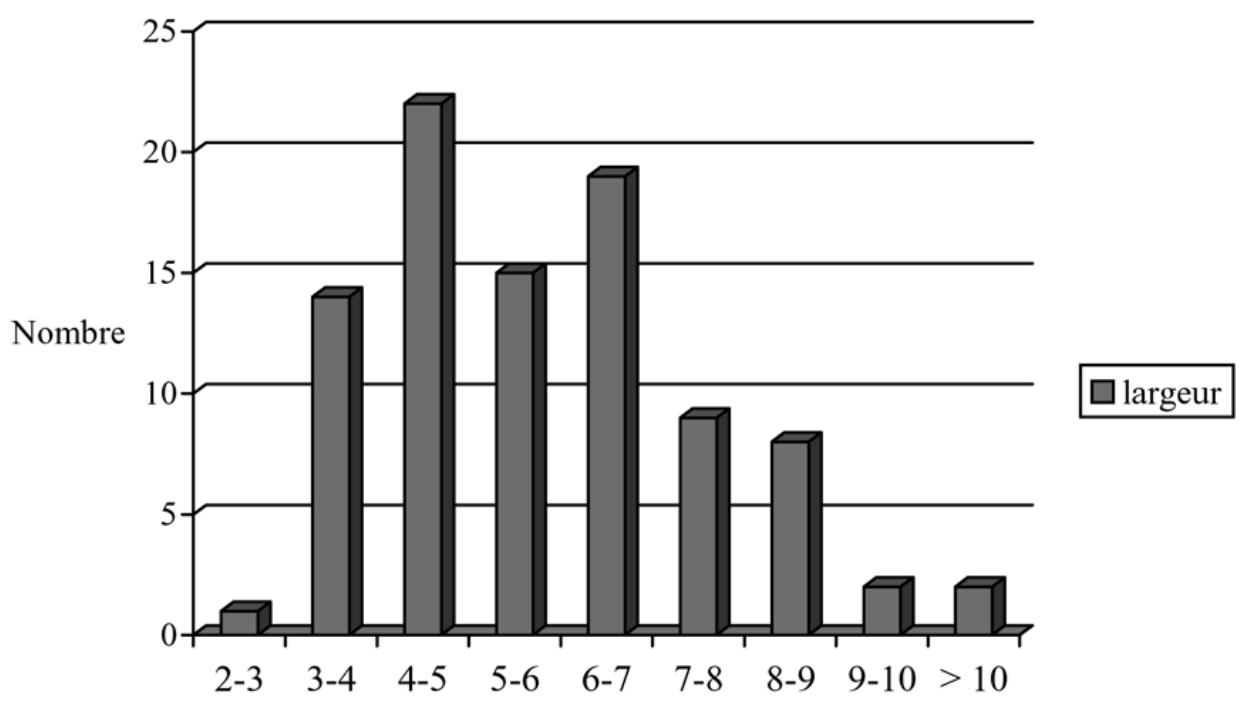

Largeur en mm

9 La petite taille de ces pièces et leur relative standardisation les rapprochent des armatures microlithiques du Mésolithique moyen (Surmely 2003) et traduit « incontestablement un registre fonctionnel très ciblé» (Soriano 1998). L'aménagement soigneux de la pointe, par des retouches directes ou croisées, révèle l'intention d'en faire des pointes acérées. Les pièces sont très majoritairement rectilignes. L'ensemble de ces caractéristiques permet de supposer qu'il s'agit de pointes de projectiles, comme l'avaient déjà suggéré de nombreux chercheurs (Praslov 1982; Praslov et Rogachev 1982 ; Cattelain 1994 et 1997 ; Soriano 1998 ; Digan 2001 ; Morala 2003).

Cette hypothèse a été soumise à l'analyse tracéologique. 


\section{L'étude tracéologique}

11 Paradoxalement l'emploi comme élément de projectile ne favorise pas la formation des traces d'utilisation. Celles-ci, que ce soit sous la forme de micro-fractures, de stries ou de zones polies, sont le résultat d'un contact intense et prolongé entre deux matériaux (Plisson et Geneste 1989 ; Fischer et al. 1984 ; Geneste et Plisson 1993). Mais l'usure de la pointe, c'est à dire de la partie en contact avec la proie, est souvent infime, à cause de l'absence de mouvement répétitif (il y a un seul impact avec la proie et un seul mouvement inverse lors de l'extraction). D'autre part le processus de fabrication de l'arme ne permet pas la formation de microtraces. L'usure, jusqu'à la partie emmanchée de l'objet, est le résultat combiné de l'impact, du mouvement du manche lui-même et, dans le cas d'outils composites, du mouvement d'extraction de ce manche, à des fins de réutilisation. Si un outil lithique est bien construit, il doit y avoir peu de mouvements du manche lui-même, donc peu de traces d'utilisation (Hays et Lucas 2001 ; Juel Jensen 1988 ; Moss 1983 ; Moss et Newcomer 1982 ; Shea 1988).

Il faut aussi considérer la question du lieu de découverte. Le site où le projectile a été trouvé est souvent le lieu où il a été recyclé. D'une manière générale, on a utilisé l'objet pour la première fois loin de l'endroit où il a été abandonné. Des pointes ou des microlithes ont pu être utilisés comme armes de chasse, mais ces mêmes pièces ont pu être rapportées jusqu'au site, pour y être recyclées sous forme d'outils à trancher (Ibanez-Estevez et Gonzalez-Uruijo 1996; Keeley 1988 ; Kimball 1989 ; Moss 1983). Ces utilisations multiples et ces recyclages rendent complexes la structure de l'objet et son identification. Quand un objet est recyclé sous forme de couteau, les mouvements de coupe éliminent les traces qui avaient été produites auparavant.

\section{Généralités}

Nous avons étudié 37 pièces provenant du site du Sire, à la recherche de microtraces qui déterminent leur fonction. Parmi ces 37 objets, 26 ont été identifiés comme des microgravettes, 6 comme des pointes à dos microlithiques, 3 comme une Gravette et 2 comme des fragments de microlithes de type indéterminé. On considère que ces objets ont été utilisés comme projectiles (Bordes 1952) mais, dans un deuxième temps, beaucoup ont pu servir de couteau (Donahue 1988 ; Harrold 1993 ; Kimball 1989). Quant aux microgravettes, elles ont pu appartenir à des outils composites ou avoir été emmanchées isolément.

14 Jusqu'ici très peu de pointes de la Gravette ont été l'objet d'une étude tracéologique. Dans sa thèse, Kimball (1989) a étudié du matériel provenant du niveau $7 \mathrm{du}$ Périgordien supérieur du Flageolet 1 . Il a analysé 32 gravettes et microgravettes et découvert que certaines avaient servi de projectile, puis, après cassure, de couteau, tandis que d'autres apparemment n'avaient été utilisées que pour la découpe de la viande.

15 L'étude de Donahue, faite en 1988 sur le site gravettien de Paglicci Cave (Italie), concernait des pointes à dos, plus petites que des pointes de La Gravette (peut-être de la taille de microgravettes). Et pourtant cette étude a montré que les pointes les plus petites ont servi d'extrémité de projectile et que les plus grosses ont été utilisées pour la découpe de matière carnée. Récemment, O’Farrell (2004) a confirmé l'utilisation de la 
majorité des Gravettes du site de Corbiac comme projectile, et ce à partir d'observations de cassures.

\section{Méthodes}

Pour ce travail nous avons utilisé les deux méthodes d'analyse tracéologique : le fort grossissement (50x à 500x) (Keeley 1980) et le faible grossissement (10x à 50x) (Odell 1977 ; Tringham et al. 1974). La méthode mise en œuvre avec faible grossissement convient à l'observation des micro et macrofractures, tandis que le fort grossissement est utilisé pour identifier et interpréter les stries et les polis.

Tout d'abord, nous avons scanné les deux faces de chacune des 17 pièces. Ensuite, nous avons imprimé ces images de telle sorte que la localisation des traces d'utilisation soit enregistrée directement dessus. Avant de passer aux analyses, tous les objets ont été plongés dans une solution nettoyante douce contenant une faible concentration de $\mathrm{NaOH}$. Cela a permis d'éliminer en douceur les résidus minéraux et organiques.

Après nettoyage, nous avons utilisé un microscope stéréo avec un zoom 10x à 50x permettant d'observer les macro-cassures et les micro-éraillures. Les traces d'utilisation ont été photographiées. Ensuite nous avons utilisé un microscope métallographique Olympus $\mathrm{BH}-2$, avec un appareil à lumière incidente et des possibilités de grossissement de 50, 100, 200 et 500 fois, permettant de faire apparaître les zones polies ou striées. En général, on observe d'abord la surface de chaque pièce, grossie 50 ou 100 fois, pour localiser les traces, avant de grossir 200 fois pour les interpréter. Nous avons fait cette identification en prenant pour référence un protocole expérimental établi par nous, et nous avons cherché des compléments ailleurs (Hays 1998 ; Hays et Lucas 2001).

\section{Microtraces caractéristiques associées à la technologie des projectiles}

19 Plusieurs sortes de traces sont associées aux projectiles. Certaines sont le résultat d'un impact, d'autres proviennent de l'opération d'emmanchement. Toutes ont des caractéristiques propres. A ce jour, de nombreux travaux expérimentaux convergent sur un fait: le type de cassure est ce qu'il y a de plus significatif pour affirmer qu'un objet a été utilisé comme pointe de projectile (par exemple Barton et Bergman 1982; Fischer et al. 1984; Albarello 1986; Nuzhnij 1989; Odell et Cowan 1986; Shea 1993; Cattelain et Perpère 1993; Cattelain 1994; Soriano 1998; O'Farrell 2004). Mais, à l'exception de O'Farrell, Soriano et Cattelain, aucune de ces études expérimentales ne pose spécifiquement le problème de l'utilisation des pointes de La Gravette et microgravettes.

Les pointes peuvent se briser dans des circonstances très variées mais certaines cassures causées par un impact se différencient de toutes les autres. Il semble qu'il y ait quatre sortes de cassures dues à l'impact d'un projectile: par flexion, en forme de burin, latérale, et par écrasement. Les cassures latérales et les écrasements peuvent être la conséquence de plusieurs autres utilisations, de phénomènes technologiques ou de phénomènes postérieurs au dépôt. Une cassure en flexion est caractéristique d'un impact de projectile quand elle a une longueur de $2 \mathrm{~mm}$ ou davantage. Elle commence à l'extrémité, au point d'impact, et se termine en cassure à «cran » ou à " charnière ». 
Ces fractures enlèvent de la matière sur les deux faces de la pièce. Il existe aussi des cassures en "vrille", plus petites. Ce sont des cassures en "cône", provenant de l'énergie cinétique résiduelle (Fischer et al. 1984). Les fractures en "burin" commencent à la pointe et continuent parallèlement à l'un des bords, se terminant par un angle de $90^{\circ}$. Ce qui en résulte a l'apparence d'une chute de burin (Fischer et al. 1984 ; Albarello 1986 ; Geneste et Plisson 1993 ; O’Farrell 2004 ; Plisson et Geneste 1989). Cela étant dit, on a remarqué que les extrémités de pointes ne se cassent pas toujours sous le choc. Des expériences ont montré que des pièces ont pu recevoir plusieurs chocs avant de se briser (Kimbal 1992).

21 Lors d'un tir, se produisent des stries et des polis tout à fait caractéristiques (Fischer et al. 1984; Geneste et Plisson 1993; Plisson et Geneste 1989). Nous avons dit précédemment que polis et stries se forment par contact direct et prolongé entre deux matériaux. Ce n'est pas le cas en ce qui concerne les projectiles car il y a seulement un impact et un mouvement pour retirer le projectile. Il peut aussi y avoir un mouvement pendant que la pointe est enfoncée dans la proie. Le poli qui se forme ainsi est atypique. Ce n'est pas le même que celui qui se forme quand un outil est utilisé pour couper ou gratter.

22 S'il y a un poli d'os, de peau ou de chair, il est discontinu. Ces types de polis sont rares. Le plus souvent, les polis ou les stries apparaissent sous la forme de bandes étroites et claires, parallèles à l'axe longitudinal de la pièce car la force de l'impact produit de petites esquilles de silex ou d'os qui ensuite sont traînées sur la surface de l'objet. On a observé expérimentalement que ces éclats adhèrent à la surface du silex (Fischer et al. 1984).

Bien évidemment, comme le souligne Albarello (1986), la nature et l'importance des traces sont fonction de plusieurs paramètres qui tiennent à la vitesse du projectile, à sa présentation sur l'impact, à la forme de l'armature, à l'homogénéité de l'assemblage formant l'ensemble du trait et surtout à la nature du corps atteint par le projectile. Les corps durs et semi-durs (os, tendons, peau épaisse) sont les plus propices à la formation de microtraces. Plusieurs auteurs soulignent que la simple rencontre avec des tissus tendres (muscles) n'est pas de nature à laisser des traces identifiables.

Les dommages causés par l'emmanchement sont aussi problématiques. « Il n'existe pas de type d'usure, simple et discrète, qu'on pourrait appeler usure du manche de l'outil. Ces traces révèlent seulement une usure de l'outil et ont peu de signification en tant que traces d'utilisation, et elles ne correspondent pas à ce qu'on peut attendre d'un frottement d'un outil contre son manche " (Cahen et al. 1979). On peut trouver à la base de la pointe des micro-fractures à cran et des polis résultant de l'emmanchement.

\section{Résultats}

L'ensemble que nous avons étudié comprend 37 pièces. Six sont entières et les 31 autres sont des fragments, majoritairement distaux (25 sur 31$)^{2}$. Sur seulement cinq des 37 pièces on peut observer des traces d'utilisation, vingt ne présentant aucune trace et douze étant trop patinées pour permettre une observation. Ces cinq pièces sont toutes considérées comme des microgravettes. Deux d'entre elles ne sont pas fragmentées et les trois autres, classées comme fragments distaux, ont de petites cassures à l'extrémité. Deux pièces ont des cassures en flexion à l'extrémité distale et une a un 
poli linéaire. Les descriptions des traces d'utilisation sont conformes à la nomenclature proposée par Fischer et al. (1984).

Les matières premières utilisées pour les trois fragments distaux (aux extrémités cassées) sont le silex blond crétacé (2 ex.) et le silex tertiaire local (1 ex). Leur longueur est d'environ $15 \mathrm{~mm}$, leur largeur est d'environ $4 \mathrm{~mm}$. A leur extrémité distale même, il y a une cassure en flexion, d'une longueur supérieure à $2 \mathrm{~mm}$. L'une se termine par un " cran », l'autre par une " charnière ». Dans deux cas, en plus de ces cassures en flexion, il y a des cassures en vrille. L'une des pièces a aussi un poli à l'extrémité de la cassure (fig. 3). Nous croyons pouvoir affirmer que ces deux cassures proviennent d'un choc dû à une projection.

Figure 3 - Fragment de microgravette, montrant une fracture terminale (A) et un poli d'impact (B). Figure 3 - Microgravette fragment showing a terminal break (A) and an impact polish (B).

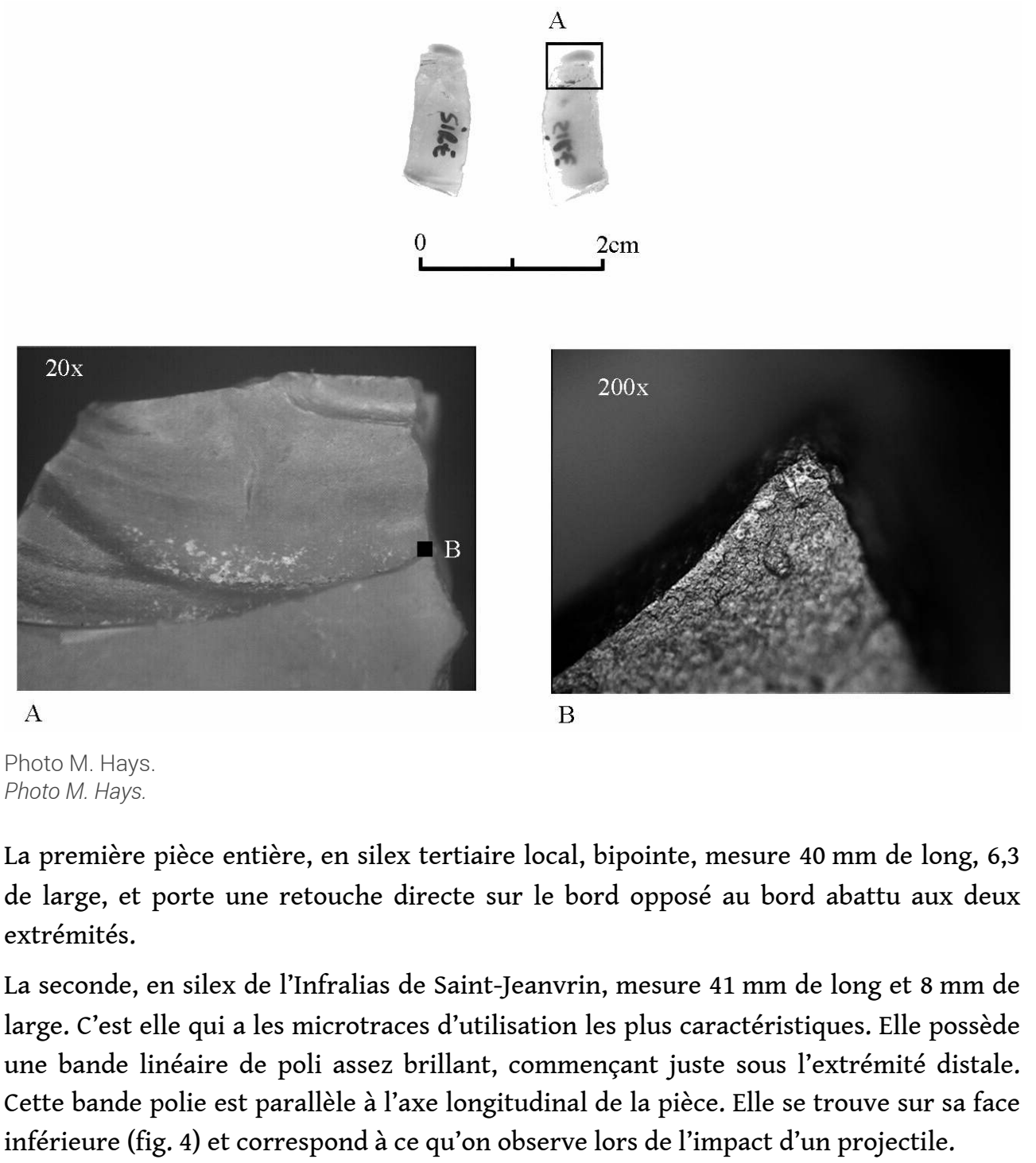


Figure 4 - Microgravette entière en silex de l'Infralias, avec poli linéaire orienté parallèlement à l'axe longitudinal de la pièce.

Figure 4 - Complete microgravette made from Infralias flint, with linear polish along the longitudinal axis of the piece.
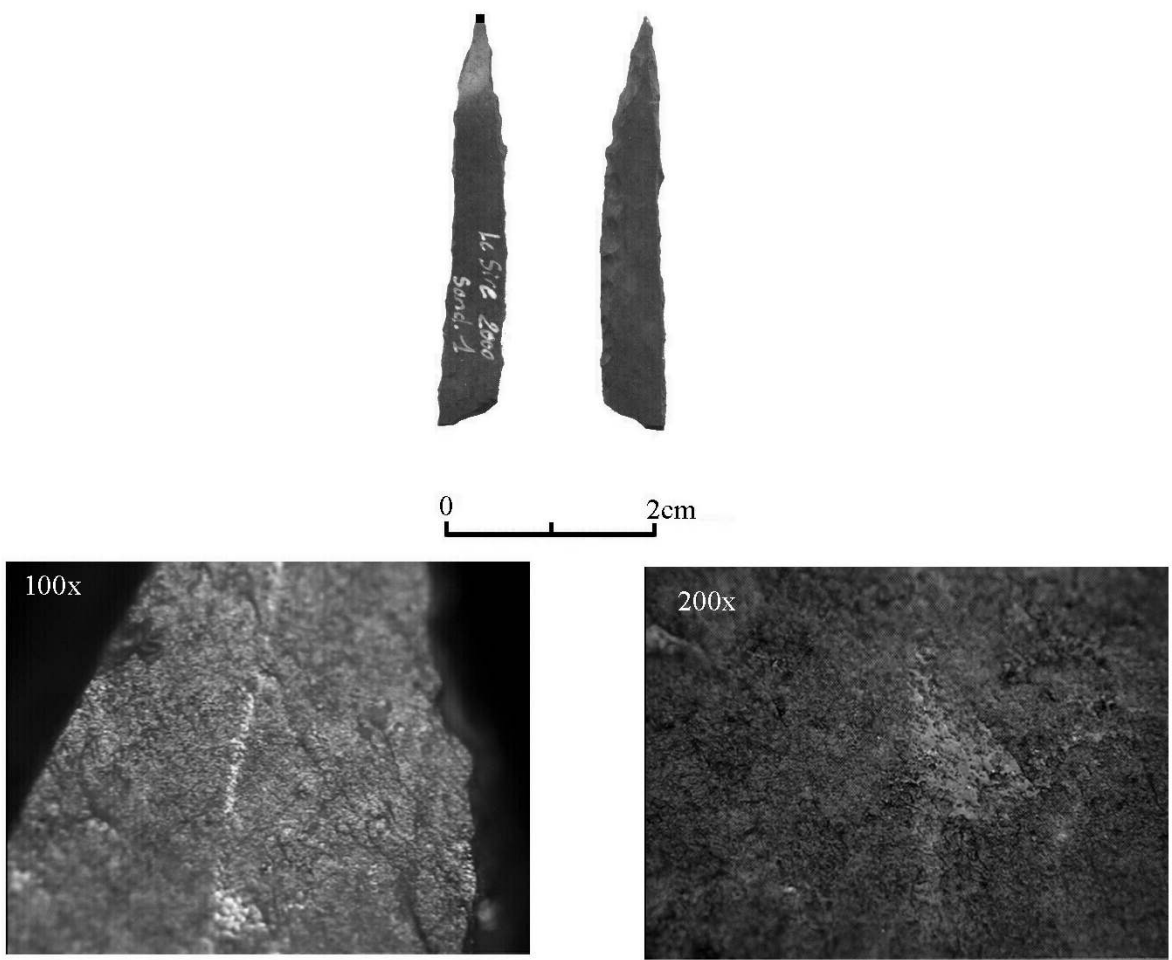

Photo M. Hays.

Photo M. Hays.

\section{Interprétations}

Bien que le nombre de pièces étudiées soit réduit et qu'il y ait une proportion importante de fragments distaux, on peut tirer plusieurs conclusions de l'analyse des pointes du gisement du Sire.

On peut d'abord évoquer l'hypothèse selon laquelle ces objets aient eu des utilisations multiples et aient été recyclés comme outils tranchants. Les travaux d'Harrold (1993) et de Keeley (1988) ont montré que certaines gravettes ont servi de couteaux, d'autres de pointes et quelques-unes ont eu les deux usages. Bien qu'ils n'aient pas séparé nettement les catégories morphofonctionnelles, il semble que les plus petites pièces aient surtout servi de pointes et les plus grosses de couteaux. Si c'est le cas, un recyclage supplémentaire pour en faire des outils tranchants peut dissimuler les traces provoquées par une projection. Mais est-ce le cas pour les microgravettes du Sire?

31 Au Sire, le recyclage des microgravettes est improbable, essentiellement en raison de leur forme. Les microgravettes ne peuvent pas avoir servi de couteaux car elles sont bien trop petites pour être utilisées seules, même munies d'un manche. Si elles avaient été les éléments d'un outil composite, elles n'auraient pas besoin d'être pointues.

C'est là qu'il est intéressant de considérer également le problème de la fragmentation. Quand une pointe est projetée et se brise lors d'un impact, en général la partie apicale 
est perdue et la base est rapportée au site pour y être réparée ou destinée à un usage secondaire. Plusieurs raisons pourraient expliquer la présence des fragments apicaux de microgravettes. A cause de la retouche de l'extrémité, on pourrait conclure en toute logique qu'ils ont été cassés lors de la fabrication et n'ont donc jamais servi. Cela pourrait être confirmé par l'absence de traces d'utilisation sur ces pièces. Elles ont pu avoir d'autres fonctions (graver, couper) et ont pu être cassées en cours d'utilisation. $\mathrm{Au}$ Sire, cette hypothèse ne tient pas car il n'y a pas de preuves sous la forme de fractures, stries ou polis qui pourraient donner à penser qu'il s'agit d'outils emmanchés servant à trancher. Enfin, Leroi-Gourhan (1983), puis Geneste et Plisson (1993) ont suggéré que les fragments apicaux ont pu être transportés dans le corps de l'animal après sa mise à mort. Dans le cas de deux fragments distaux, il y a des traces d'utilisation.

L'étude tracéologique confirme donc l'idée initiale. Toutes les traces retrouvées sur les pièces correspondent à une utilisation comme pointe de projectile. Aucun indice n'a pu être trouvé en faveur d'un usage comme barbelure latérale ou bien comme couteau, ce qui est logique, vu l'appointement et la petite taille de ces éléments. Les outils présentant des traces forment un pourcentage assez faible du corpus étudié ( 5 sur 37, soit moins de $15 \%$ ). Mais il ne faut pas oublier que 12 pièces étaient trop patinées pour permettre une analyse. Une proportion importante des pièces $(55 \%)$ ne montre pas de traces, ce qui est la règle générale pour tous les corpus étudiés ${ }^{3}$ (Philibert 2000). Mais, comme nous l'avons vu plus haut, ceci n'est pas incompatible avec leur statut de pointe axiale, bien au contraire. On peut penser que certaines n'ont pas été utilisées. D'autres enfin ont pu servir mais la simple rencontre fugace de tissus mous n'a pas laissé de traces apparentes sur l'élément en silex.

\section{Arc ou propulseur?}

Beaucoup d'études ont été consacrées à la question de l'identification du mode de propulsion (Cattelain 1994 et 1997 ; Geneste et Plisson 1993 ; Lansac 2004). Des études antérieures, menées sur des pointes de plus grandes dimensions et de poids nettement supérieur (pointes de la Gravette ou pointes à cran solutréennes), ont montré la possibilité d'utilisation de deux modes de propulsion impliquant deux types de projectiles : flèche d'arc et sagaie lancée au propulseur ou à la main, sans qu'il soit possible de trancher entre les deux choix (Plisson et Geneste 1989; Carrère 1990; Cattelain et Perpère 1993) ${ }^{4}$.

Dans le cas des microgravettes et petites pointes à dos, le problème est bien différent. En effet, ces armatures sont de taille et de poids bien inférieurs à ceux des grosses armatures citées plus haut. La possibilité de leur emploi sur une sagaie classique paraît fort improbable.

Les mesures effectuées par P. Cattelain sur des propulseurs ethnographiques montrent en effet que la largeur des hampes n'est jamais inférieure à $15 \mathrm{~mm}$ et que le poids des projectiles n'est jamais inférieur à $5 \mathrm{~g}$ (Cattelain 1994).

Pour tenter de progresser dans la voie de la discrimination entre les deux types de projectiles, nous avons réalisé l'étude d'un échantillon d'environ 600 flèches provenant des collections ethnographiques de deux musées: le musée du Périgord à Périgueux (Dordogne) et le musée d'histoire naturelle de La Rochelle (Charente-Maritime) (fig. 5). Ces flèches proviennent de trois continents : Amérique du Nord et du Sud, Asie du Sud- 
Est et Afrique. Cent quatre vingt dix-neuf pièces ont fait l'objet d'une fiche détaillée, avec les mesures de leur longueur, de leur poids, du diamètre de la hampe, de la nature des composants utilisés, etc. On décompte 151 pointes en fer, 30 en bois et épines, 5 en dents, 4 en coquillage, 4 en ivoire et bois de cervidé et deux en silex. Cette étude fera l'objet d'une publication prochaine.

Figure 5 - Flèche à pointe en fer des Indiens commanches (ref. H 2096).

Figure 5 - Iron-head arrow of the Comanches Indians (ref. H 2096).

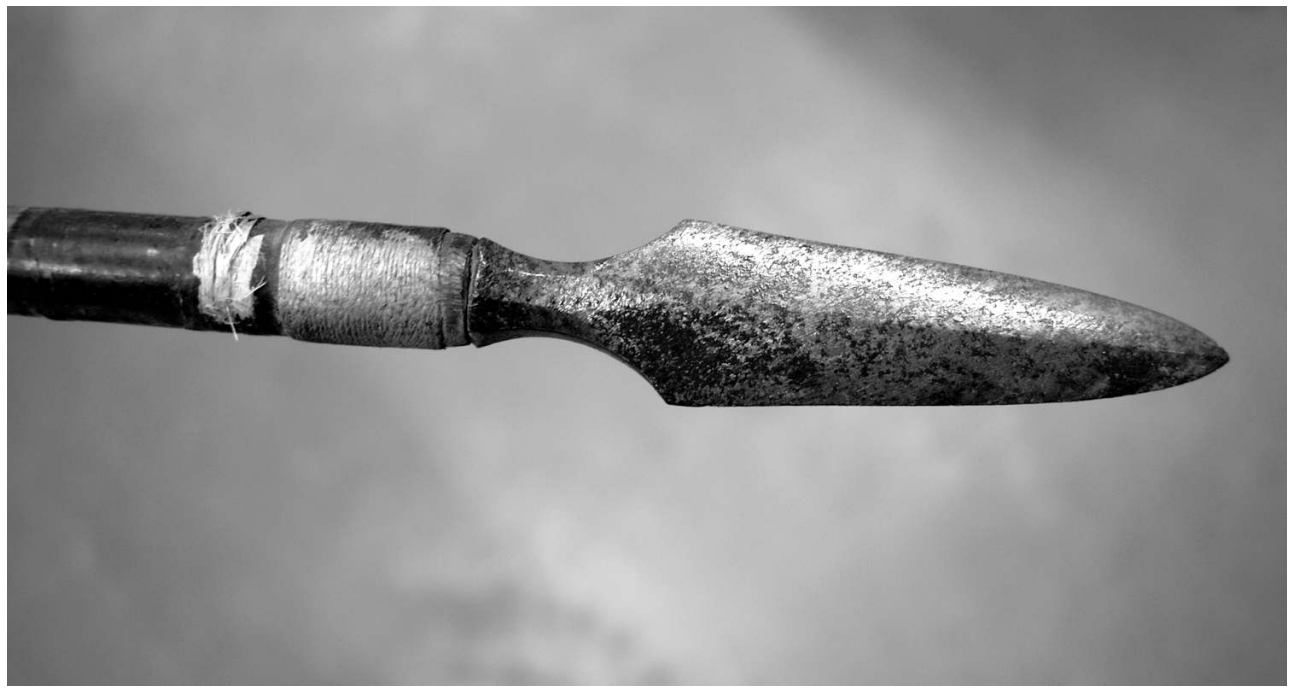

Diamètre hamper : $8,5 \mathrm{~mm}$

Diametre of the shaft : $8.5 \mathrm{~mm}$

Musée d'Histoire naturelle de La Rochelle.

Museum of natural History of La Rochelle (France).

Pour ce qui est de la question qui nous intéresse, l'observation essentielle est que la largeur de la pointe est presque toujours supérieure à celle de la hampe (93\% des cas). Si on élimine les grandes flèches de plus de $120 \mathrm{~cm}$ qui proviennent d'îles et étaient probablement destinées à la pêche (îles Salomon, Nouvelles Hébrides), cette valeur monte à 95,4 \%. Cela s'explique fort logiquement par le fait que le pouvoir vulnérant du projectile (et non sa simple valeur balistique) repose d'abord sur sa capacité de pénétration, qui serait nettement amoindrie si la hampe avait une largeur supérieure à celle de la pointe. Toutes les études détaillées portant sur des armatures de différentes périodes, allant du Gravettien au Mésolithique (Soriano 1998 ; Digan 2001 ; Montoya 2002 ; Surmely 2003) ont mis en évidence la recherche de la calibration de la largeur, qui est toujours le paramètre morphologique le mieux maîtrisé ${ }^{5}$, alors que les longueur, épaisseur, poids sont plus variables.

Le diamètre ${ }^{6}$ des hampes des pièces ethnographiques est en moyenne de 7,4 $\mathrm{mm}$ mais cette valeur représente en effet la moyenne de deux groupes nettement séparés. Les flèches à pointes en fer, lourdes et longues, ont des hampes à diamètre plutôt important (8 à $10 \mathrm{~mm}$ ), alors que les flèches "traditionnelles ", à pointes en silex, sont de dimensions plus modestes $(5,7$ et $6,5 \mathrm{~mm})$. Les flèches épipaléolithiques et mésolithiques ont un diamètre variant de 5 à 10 mm (Rust 1943 ; Rozoy 1978 ; Cattelain 1994). Enfin, il est à noter que les formules composites, associant deux pièces en tête de projectile sont rarissimes. Nous avons pu observer quelques rares dispositifs groupant une pointe et une barbelure latérale mais les montages comportant deux pointes 
accolées sont totalement absents. Cela apparait logique compte tenu de la difficulté qu'il y aurait à trouver un dispositif de fixation solide pour deux pointes accolées dont les bords ne peuvent pas être exactement parallèles.

Ces observations ethnographiques confortent l'idée du rejet de l'emploi de petites pointes sur des hampes de sagaie dont le diamètre est nettement supérieur à celui des hampes de flèches. Il est inutile de penser à un amincissement important de la partie distale de la hampe, ayant pu favoriser l'emploi de petites pointes. En effet, cet amincissement ne peut pas être très important, sous peine de fragiliser la hampe et de la déséquilibrer. Cela a pu être vérifié sur notre corpus ethnographique.

On pourrait penser à un dispositif associant deux pointes, étroitement accolées ou bien disposées de façon légèrement oblique, afin que leurs pointes se rejoignent. Les largeurs des deux pièces s'additionneraient alors pour former une pointe large, utilisable sur une hampe de diamètre respectable. Cette idée nous paraît toutefois peu plausible. En effet, nous avons vu que ce type d'emmanchement est absent du corpus ethnographique que nous avons pu étudier. Cela s'explique par le fait que nous voyons mal l'utilité d'un tel assemblage, nécessitant un dispositif de fixation assez complexe et probablement plutôt fragile, par rapport à l'utilisation d'une simple pointe de largeur plus grande, du type Gravette, fléchette ou même Font-Robert, alors même que ces pointes de grande taille sont par ailleurs présentes dans l'assemblage lithique du gisement du Sire.

L'hypothèse de pointes destinées à des sarbacanes parait à rejeter formellement car l'examen de quelques armes de ce type nous a montré qu'elles étaient toujours associées à des flèches minuscules, sans pointe ajoutée et dont le poids total ne dépasse pas $2 \mathrm{~g}$.

En conclusion, l'hypothèse de la flèche d'arc nous semble donc la seule à retenir et va dans le sens des théories déjà émises à ce sujet. La largeur moyenne des microgravettes et pointes correspond à peu près à la largeur des hampes des flèches ethnographiques à pointe en silex et de certaines flèches épipaléolithiques et mésolithiques. Compte tenu de la largeur des microgravettes, on peut penser qu'il s'agissait de flèches à section assez réduite, correspondant probablement à des arcs peu puissants.

Reste évidemment le problème posé par les plus minces des microgravettes, dont la largeur paraît vraiment faible, si l'on retient l'idée que le diamètre de la hampe ne devait pas être supérieur. Leur forme identique aux autres exclut l'idée selon laquelle elles auraient pu être placées en position latérale (barbelures) et rend improbable un autre usage (par exemple micro-perçoir comme à Pincevent ; Moss et Newcomer 1982). Ce point demeure toutefois à éclaircir.

Bien évidemment, cette hypothèse d'utilisation de l'arc avec des pointes de type microgravette, n'exclut pas l'idée de l'emploi parallèle de la sagaie lancée au propulseur qui aurait pu recevoir des pointes plus volumineuses comme les Gravette, Font-Robert et fléchettes.

\section{Conclusions générales}

Notre travail, malgré le petit nombre de pièces étudiées, a permis d'arriver à la conclusion que les microgravettes, outils abondants dans le site gravettien ancien du Sire, pouvaient être identifiées comme des pointes armant des projectiles. Cela est en 
accord avec la fonction du gisement qui semble correspondre à un site d'abattage spécialisé (chasse au cheval principalement).

Ces armatures microlithiques ne pouvaient convenir à un projectile lourd et imposant du type de ceux lancés à la main ou avec un propulseur. Inversement, les pièces sont beaucoup trop grosses pour avoir armé une sarbacane, en supposant qu'une arme de ce type ait pu être façonnée par les hommes du Gravettien. Par élimination et en accord avec l'observation de pièces ethnographiques, on est en droit de supposer que ces pièces ont pu être des pointes de flèches d'arc. Ceci va dans le sens d'études antérieures réalisées sur d'autres pièces plus lourdes, telles que des pointes de la Gravette ou des pointes à cran qui laissaient supposer l'emploi possible de l'arc durant le Paléolithique supérieur. On peut donc penser que l'arc était connu et largement utilisé au Gravettien ancien. Considérant la présence de pièces morphologiquement très proches (telles que certaines lamelles ou pointes de Font-Yves) dans des contextes chronologiques antérieurs, il est même envisageable que cette arme ait pu être inventée dès les débuts du Paléolithique supérieur (O'Farrell 2004 et sous presse). Reste toutefois que les pièces ayant le plus petit gabarit semblent bien petites pour armer des flèches de taille standard.

\section{BIBLIOGRAPHIE}

ALBARELLO B. 1986 - Sur l'usage des microlithes comme armatures de projectiles. Revue archéologique du Centre de la France, t. 25, fasc. 2, p. 127-143.

BARTON R.-N.-E. et BERGMAN C.-A. 1982 - Hunters at Hengistbury: some evidence from experimental archaeology. World Archaeology, 14, p. 237-248.

BORDES F. 1952 - A propos des outils à bord abattu, quelques remarques. Bulletin de la Société Préhistorique Française, t. 49, p. 645-647.

CAHEN D., KEELEY L.-H. et VAN NOTEN F.-L. 1979 - Stone Tools, Toolkits, and Human Behavior in Prehistory. Current Anthropology, 20, p. 661-683.

CARRÈRE P. 1990 - Contribution de la balistique au perfectionnement des études technofonctionnelles des pointes de projectiles préhistoriques. Paléo, n² 2, p. 167-190.

CATTELAIN P. 1994 - La chasse au paléolithique supérieur. Arc ou propulseur, ou les deux ? ArchéoSitula, t. 21-24, p. 5-26.

CATTELAIN P. 1997 - Hunting during the upper Palaeolithic : bow, spearthrower or both? In KNECHT (H.) - Projectile technology. Plenium press corporation, New-York, 408 p.

CATTELAIN P. et PERPERE M. 1993 - Tir expérimental de sagaies et de flèches emmanchées de pointes de La Gravette. Archéo-Situla, t. 17-20, p. 3-25.

DEMARS P.-Y. et LAURENT P. 1989 - Types d'outils lithiques du Paléolithique supérieur en Europe. Cahiers du Quaternaire ${ }^{\circ} 14$, éditions du CNRS, Paris, $178 \mathrm{p}$. 
DIGAN M. 2001 - Le gisement gravettien de la vigne-Brun (Loire) : première étude de l'industrie lithique de l'unité KL19. Thèse de doctorat de l'université de Lille I, 2 vol., 465 p.

DONAHUE R. 1988 - Microwear Analysis and Site Function of Paglicci Cave, Level 4A. World Archaeology, 19, p. 357-375.

FISCHER A., VEMMING-HANSEN P. et RASMUSSEN P. 1984 - Macro and micro wear on lithic projectile points. Experimental results and prehistoric examples. Journal of Danish Archaeology, n 3, p. 19-46. GENESTE J.-M. et PLISSON H. 1993 - Hunting technologies and human behavior : Lithic analysis of Solutrean shouldered points. InBefore Lascaux: The Complex Record of the Early Upper Paleolithic, edited by H. Knecht, A. Pike-Tay, and R. White, p. 117-135. Boca Raton : CRC Press.

HARROLD F. 1993 - Variability and Function Among Gravette Points from Southwestern France. In Hunting and Animal Exploitation in the Later Paleolithic and Mesolithic of Eurasia, edited by G. Larsen Peterkin, H. Bricker, and P. Mellars, pp. 69-79. Archaeological Papers of the American Anthropological Association.

HAYS M. 1998 - A Functional Analysis of the Magdalenian Assemblage From Grotte XVI (Dordogne, France). Unpublished Ph.D. dissertation, Department of Anthropology, University of Tennessee, Knoxville.

HAYS M. et LUCAS G. 2001 - Experimental Investigations of Aurignacian Dufour Bladelets. In Questioning the Answers: Resolving Fundamental Problems of the Early Upper Paleolithic, edited by M. Hays and P. Thacker, p. 109-116. BAR International Series 1005. Oxford, British Archaeological Reports.

IBANEZ-ESTEVEZ J.-J. et GONZALEZ-URUIJO J.-E. 1996 - From tool use to site function, use wear analysis in some final upper Paleolithic sites in the Basque country. Oxford, British Archaeological Reports International Series, 658.

JUEL-JENSEN H. 1988 - Functional Analysis of Prehistoric Flint Tools by High Power Microscopy : A Review of West European Research. Journal of World Prehistory, 2, p. 53-88.

KEELEY L.H. 1988 - Experimental Determination of Stone Tool Use: A Microwear Analysis. Chicago, University of Chicago Press.

KIMBALL L. 1989 - Planning and Functional Variability in the Upper Paleolithic: Microwear Analysis of Upper Perigordian Tools from Le Flageolet I. Ph.D. dissertation, Northwestern University. Ann Arbor, University Microfilms International.

KIMBALL L. 1992 - Microwear Analysis of the Functions of Projectile Points and Unifacial Tools. In Archaeological Data Recovery for Transcontinental Gas Pipe Line Corporations 6.79-mile Leidy Natural gas Pipeline Expansion, Padula Site (36-Nm-15), Northhampton County Pennsylvania, edited by C.A. Bergman and J.F. Doershuk, pp. 238-288. Technical Report Submitted to Transcontinental Gas Pipe Line Corporation, Houston, TX.

LADIER E. (dir.) 2003 - Les pointes à cran dans les industries lithiques du Paléolithique supérieur récent, de l'oscillation de Lascaux à l'oscillation de Bölling. Préhistoire du Sud-Ouest, supplément n 6, 165 p.

LANSAC J.-P. 2004 -Un cadre chronologique pour l'utilisation du propulseur et de l'arc durant le Paléolithique supérieur. Bulletin des Chercheurs de la Wallonie, 43, p. 29-36.

LEROI-GOURHAN A. 1983 - Une tête de sagaie à armature de lamelles de silex à Pincevent (Seine-etMarne). Bulletin de la Société Préhistorique Française, t. 85, p. 154. 
MONTOYA C. 2002 - Les pointes à dos épigravettiennes de Saint-Antoine-Vitrolles (Hautes-Alpes) : diversité typologique ou homogénéité conceptuelle? Bulletin de la Société Préhistorique Française, t. $99, \mathrm{n}^{\circ} 2$, p. $275-288$.

MORALA A. 2003 - Discussion sur le thème des microgravettes. In LADIER E. (dir.) - Les pointes à cran dans les industries lithiques du Paléolithique supérieur récent, de l'oscillation de Lascaux à l'oscillation de Bölling. Préhistoire du Sud-Ouest, supplément nº 6, p. 161.

MOSS E.-H. 1983 - The Functional Analysis of Flint Implements: Pincevent and Pont d'Ambon : two Case Studies from the French Final Paleolithic. Oxford, British Archaeological Reports International Series, 177.

MOSS E.-H. et NEWCOMER M. 1982 - Reconstruction of Tool Use at Pincevent: Microwear and Experiments. Studia Praehistorica Belgica, 2, p. 289-312.

NESPOULET R. 1996 - Le Périgordien VI de l'abri Pataud (Les-Eyzies-de-Tayac, Dordogne). Étude technologique et typologique de l'industrie lithique de la couche 3. Thèse de doctorat du Muséum national d'Histoire naturelle, 260 p., dact

NUZHNYJ D. 1989 - L'utilisation des microlithes géométriques et non géométriques comme armatures de projectiles. Bulletin de la Société Préhistorique Française 86, p. 89-96.

ODELL G. 1977 - The Application of Micro-Wear Analysis to the Lithic Component of an Entire Prehistoric Settlemen t: Methods, Problems, and Functional Reconstructions. Ph.D. dissertation, Department of Anthropology, Harvard University, Cambridge, Massachusetts. Ann Arbor : University Microfilms, No. 77-27, 354.

ODELL G. et COWAN F. 1986 - Experiments with Spears and Arrows on Animal Targets. Journal of Field Archaeology, 13, p. 195-212.

O'FARRELL M. 2004 - Les pointes de la Gravette de Corbiac (Dordogne) et considérations sur la chasse au Paléolithique supérieur ancien. In Approches Fonctionnelles en Préhistoire. Actes du $X X V^{\mathrm{e}}$ Congrès Préhistorique de France, p. 121-138

O'FARRELL M. Sous presse - Étude préliminaire des éléments d'armature lithique de l'Aurignacien ancien de Brassempouy. In LEBRUN-RICALENS F. (dir.) Productions lamellaires attribués à l'Aurignacien : chaînes opératoires et perspectives technoculturelles. XIV ${ }^{\mathrm{e}}$ congrès de l'UISPP, Paris-Luxembourg.

PHILIBERT S. 2000 - Analyse fonctionnelle des occupations du Magdalénien des grottes Jean-Pierre 1 et 2 à Saint-Thibaud-de-Couz (Savoie). In TILLET G. (dir.) - Les paléoalpins, hommage à Pierre Bintz, Géologie alpine, $\mathrm{n}^{\circ}$ 31, p. 147-154.

PLISSON H. et GENESTE J.-M. 1989 - Analyse technologique des pointes à cran solutréennes du Placard (Charente), du Fourneau-du-Diable, du Pech de la Boissière et de Combe-Saunière (Dordogne). Paléo, n 1, p. 65-106.

PRASLOV N.-D. 1982 - On the time on the invention of the bow. XI Congrès de l'INQUA (résumés), vol. 2, Moscou.

PRASLOV N.-D. et ROGACHEV A.-N. 1982 - The Paleolithic of the Kostenki-Borshevo. Rayon on the Don 1879-1979. Leningrad, Nauka.

ROzoY J.-G. 1978 - Les derniers chasseurs. L'épipaléolithique en France et en Belgique. Essai de synthèse. Charleville-Mézières, Mémoires de la société Archéologique champenoise 1, 1500 p.

RUST M. 1943 - Die alt- und mittelsteinzeitlichen Funde von Stellmoor. Neumünster-in-Holstein, Karl Wachholz Verlag, 242 p, 107 fig. 
SHEA J. 1988 - Spear Points from the Middle Paleolithic of the Levant. Journal of Field Archaeology, 15 , p. 441-450.

SHEA J. 1993 - Lithic Use-Wear Evidence for Hunting by Neandertals and Early Modern Humans from the Levantine Mousterian. InHunting and Animal Exploitation in the Later Paleolithic and Mesolithic of Eurasia, edited by G. Larsen Peterkin, H. Bricker, and P. Mellars, p. 189-198. Archaeological Papers of the American Anthropological Association.

SONNEVILLE-BORDES D. et PERROT J. 1956 - Lexique typologique du paléolithique supérieur (suite et fin) - V Outillage à bord abattu - VI Pièces tronquées - VII Lames retouchées - VIII Pièces variées - IX Outillage lamellaire. Pointe azilienne. Bulletin de la Société Préhistorique Française, t. 53, $\mathrm{n}^{\circ} 9$, p. 547-559.

SORIANO S. 1998 - Les microgravettes du Périgordien de Rabier à Lanquais (Dordogne). Analyse technologique fonctionnelle. Gallia Préhistoire, t. 40, p. 75-94.

SURMELy F. (dir.) 2003 - Le site mésolithique des Baraquettes (Velzic, Cantal) et le peuplement de la moyenne montagne cantalienne, des origines à la fin du Mésolithique. Mémoire de la Société préhistorique française, t. 32, $282 \mathrm{p}$.

SURMEly F., ALIX Ph., COSTAMAgNo S., DANIEL P., HAYS M., MURAT R., RENARD R., VIRMONT J. et TEXIER J.-P. 2003 - Découverte d'un gisement du gravettien ancien au lieu-dit Le Sire, commune de Mirefleurs, Puy-de-Dôme. Bulletin de la Société Préhistorique Française, t. 100, p. 29-39.

SURMELY F., COSTAMAGNO S., HAYS M., PASTY J.-F. et ALIX Ph. Soumis - le Gravettien et le Protomagdalénien en Auvergne. In : Rigaud J.-Ph. (dir.) - Entités régionales d'une paléoculture européenne : le Gravettien. Actes de la table ronde internationale des Eyzies-de-Tayac, 2004, Paléo. SURMELY F. et PASTY J.-F. 2003 - Les importations de silex en Auvergne durant la préhistoire. Actes de la Table ronde internationale d'Aurillac «les matières premières lithiques en préhistoire ». Supplément à Préhistoire du Sud-Ouest, p. 327-336.

TRINGHAM R., COOPER G., ODELL G., VOYTEK B. et WHITMAN A. 1974 - Experimentation in the Formation of Edge Damage : A new Approach to Lithic Analysis. Journal of Field Archaeology, p. 171-196.

VALENTIN B. 2003 - discussion sur le thème des pointes à cran. In LADIER E. (dir.) - Les pointes à cran dans les industries lithiques du Paléolithique supérieur récent, de l'oscillation de Lascaux à l'oscillation de Bölling. Préhistoire du Sud-Ouest, supplément nº, p. 160-161.

\section{NOTES}

1. Les microgravettes étant des pointes à dos rectiligne, la largeur maximale de la pièce n'excède pas beaucoup celle de la partie distale (si celle-ci ne se limite pas à l'extrémité de la pointe).

2. Le choix préférentiel des fragments distaux au détriment des fragments mésiaux est volontaire, dans le but de privilégier les pièces offrant les plus fortes chances de présenter des traces d'utilisation.

3. Par exemple, l'examen de 89 pointes à dos magdaléniennes du gisement de Saint-Thibaud-deCouz n'a permis d'observer des traces que sur seulement 6 pièces (Philibert 2000).

4. L'échantillon considéré par Cattelain et Perpère correspond à des pièces dont les largeurs vont de 2 à $25 \mathrm{~mm}$, c'est-à-dire, à des Gravettes mais aussi à des microgravettes. Quant à l'expérimentation de Soriano, elle semble n'avoir considéré que des Gravettes, au vu des figures reproduites dans l'article et de la largeur des hampes utilisées. 
5. Le succès rencontré par les armatures à cran et à pédoncule, durant le PaléolithiqueMésolithique (Ladier 2003 ; Valentin 2003) et surtout le Néolithique s'explique assurément par la possibilité de disjoindre la largeur de la partie destinée à l'emmanchement de la largeur de la partie utile de l'armature, ce qui permet un meilleur calibrage de l'armature.

6. La valeur retenue est celle de la partie distale de la hampe, au contact de la pointe. La largeur de la partie médiane peut être légèrement plus importante.

\section{RÉSUMÉS}

La fouille en cours du gisement gravettien ancien du Sire (Mirefleurs, Puy-de-Dôme, France), daté des environs de $30000 \mathrm{BP}$, a révélé une abondance d'armatures microlithiques parmi lesquelles dominent les microgravettes et les pointes à dos. Une analyse tracéologique de 37 pièces, couplée à une étude comparative de 600 objets ethnographiques, permet d'émettre l'hypothèse que ces pointes constituaient des armatures de flèches, lancées avec un arc. L'utilisation de l'arc à cette période, déjà envisagée par plusieurs chercheurs, est donc très probable.

The excavations that are being carried out on the Early Gravettian site of Sire (Mirefleurs, Puy de Dôme, France), which has been dated back to around 30,000 BP, have produced a large quantity of microlithic blades and heads with a high proportion of microgravettes and hacked points. The microwear analysis of 17 objects, together with a comparative study on 600 ethnographic artifacts, enables us to venture theopinion that thosepoints were arrowheads shot from a bow. Some researchers have already thought that the bow may have been used as early as that period: now it appears to be highly probable.

Early Gravettian, Perigordian, Auvergne, Upper Palaeolithic, bow, arrow

\section{INDEX}

Mots-clés : arc, Auvergne, flèche, Gravettien ancien, Limagne, Paléolithique supérieur, Périgordien

\section{AUTEURS}

\section{MAUREEN HAYS}

College of Charleston, dept of Anthropology - 19 Saint-Philips Street SC 29424 Charleston - EtatsUnis. haysm@cofc.edu

\section{FRÉDÉRIC SURMELY}

Géolab - UMR 6042 du CNRS - Maison de la Recherche, 4, rue Ledru 63000 Clermont-Ferrand. frederic.surmely@club-internet.fr 